\title{
Numerical study of the effect of trailing edge bluntness on highly turbulent hydrofoil flow
}

\author{
T. Do* L. Chen ${ }^{\dagger} \quad$ J. Tu ${ }^{\ddagger}$
}

(received 16 January 2006; revised 18 April 2007)

\begin{abstract}
Turbulent trailing edge flow over control surfaces continues to be of interest to many designers and researchers alike due to the associated complex and adverse phenomena. In this study a two dimensional NACA0015 airfoil was modified at the trailing edge by vertically 'cutting' at various locations along the chord axis thereby changing the Reynolds number based on the trailing edge height, while keeping the chord-length based Reynolds number fixed at 3.49 million. The numerical results show the bluntness parameter at onset of vortex shedding agree well with published data for sections having a blunt trailing
\end{abstract}

*School of Aerospace, Mechanical and Manufacturing Engineering, RMIT University, Australia.

${ }^{\dagger}$ Maritime Platforms Division, DSTO Melbourne, Australia. mailto:li.chen@dsto.defence.gov. au

${ }^{\ddagger}$ School of Aerospace, Mechanical and Manufacturing Engineering, RMIT University, Australia. mailto: jiyuan.tu@rmit.edu.au

See http://anziamj.austms.org.au/V47EMAC2005/Do for this article, (c) Austral. Mathematical Soc. 2007. Published July 13, 2007. ISSN 1446-8735 
edge. A parametric study suggests that increasing the trailing edge height tends to reduce the frequency of vortex shedding. Also, the variational trend of wake formation lengths with increasing trailing edge Reynolds number resembles that of flow past a circular cylinder. The Strouhal number for this blunt section is approximately 0.09 .

\section{Contents}

1 Introduction

C823

2 Numerical Method

C825

3 Results and Discussion

C828

4 Conclusion

C837

References

C838

\section{Introduction}

Prediction of hydro/aero-acoustics associated with highly turbulent trailing edge flows of lifting surfaces is important for designing quiet machinery. Extensive investigations conducted by many researchers in the field have shown that these flow induced sounds and vibrations are usually associated with some form of flow unsteadiness, generating broadband and tonal noise. Broadband noise is caused by freestream turbulence and boundary layer turbulence, which affects the local pressure field due to the random mixing nature of the chaotic flow. Distinct tonal noise involves prominent pressure fluctuations due to periodic flow dynamics such as shedding of vortices from the trailing edge surfaces of the lifting body. 
The occurrence of vortex shedding from a lifting surface is largely dependent on the Reynolds number, Re, and the trailing edge geometric profile or the bluntness parameter which is characterised by $h / \delta^{*}[1]$, where $h$ is the height of the trailing edge and $\delta^{*}$ is the displacement thickness taken at the trailing edge. For high Re $\left(>2 \times 10^{6}\right)$ flows over submerged rigid and perfectly sharp trailing edge lifting bodies, laminar boundary layers normally develop along the fore section due to the viscous shear stress between the fluid and the solid boundary. These laminar boundary layers consequently transition into highly turbulent boundary layers enveloping the entire aft section. At low angles of attack, the presence of the sharp trailing edge allows smooth convergence of the upper and lower surface flows. Modifications of the trailing edge geometry from its inherent sharpness can alter the local boundary layer flow characteristics, leading to vortex shedding at the trailing edge. This results in substantial changes in the hydrodynamic and hydroacoustic properties of the wake in the immediate vicinity of the lifting surface [2].

Limitations on current technology in manufacturing lifting surfaces make it difficult to produce a perfectly sharp trailing edge, resulting in bluntedged lifting surfaces. Non-sharp trailing edge profiles are susceptible to flow separation and vortex shedding causing acoustic emission and structural vibration. This investigation therefore aims to provide further insights for designers regarding the influence of trailing edge geometry on the generation of lift, drag, the mechanisms associated with vortex shedding and the characteristics of the wake structure using the growingly popular and cost efficient numerical methods.

It is generally well known that no universal turbulence model exists that can practically analyse all flow situations. For instance, Direct Numerical Simulation (DNS) solves the Navier-Stokes equations directly and Large Eddy Simulation (LES) computes large scale eddy structures while modelling the smaller ones, both require significant computing resources despite the ever advancing and increasing processing power. Thus the Reynolds Averaged 


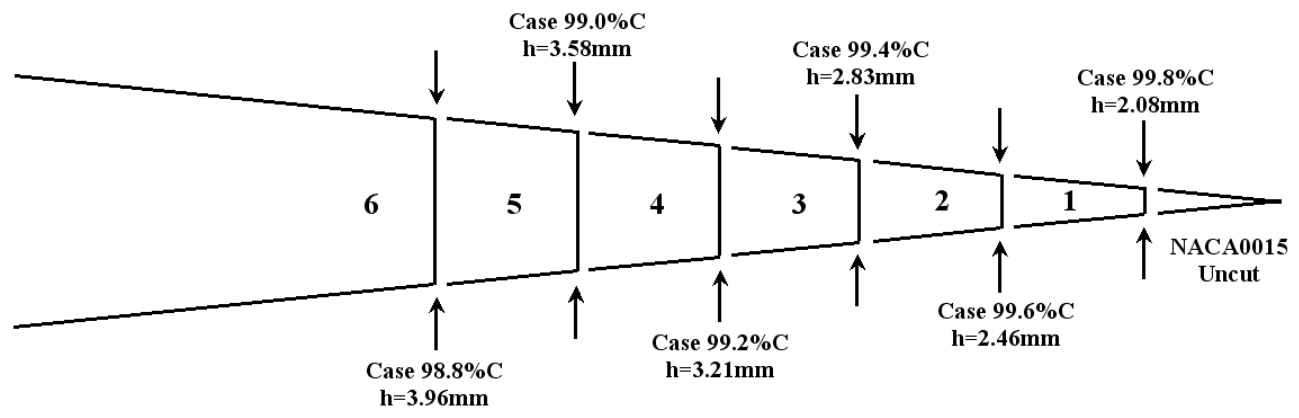

FiguRE 1: Schematic modification of trailing edges.

Navier-Stokes equations (RANS) remain the state-of-the art in the industry for analysing high Reynolds number unsteady flows [3].

Based on the assessments of the turbulence models available in the commercial software FLUENT 6.0 by Mulvany et al. [4], and the numerical prediction method by Ang et al. [5], this preliminary numerical investigation focused on determining the wake characteristics and mechanisms associated with the generation of tonal noise in high Reynolds number flows over a twodimensional airfoil section with varying degrees of trailing edge bluntness. The effects of changing the trailing edge height on the wake structure dimensions were investigated and the bluntness parameter at which vortex shedding occurs was numerically computed. Finally, the inherent Strouhal number for this section was determined and compared with published experimental data from Blake [1] and Brooks \& Hodgeson [6].

\section{Numerical Method}

In this study, a two-dimensional NACA0015 section, commonly used as control surfaces, was chosen for numerical computation and analysis. The blunt trailing edge was modelled in the worst case scenario by placing vertical 
'cuts' along the trailing edge of the originally sharp edged NACA0015 section as shown in Figure 1. In total, six cases were studied with the first case having the cut at $99.8 \% \mathrm{C}$, where $C$ is the chord length of the original NACA0015 section and the last case with the most extreme cut located at $98.8 \% \mathrm{C}$. All cases had the two-dimensional section aligned parallel to the direction of the oncoming flow with a freestream velocity, $U_{\infty}$, of $5 \mathrm{~ms}^{-1}$. Properties of water were chosen to model the working medium and the corresponding Reynolds number based on the chord length was $\mathrm{Re}=3.49 \times 10^{6}$. Thus the main variable parameter was the Reynolds number based on the trailing edge height, $\mathrm{Re}_{h}$.

The two-dimensional section was created based on the chord dimension of $C=540 \mathrm{~mm}$, which was embedded in a rectangular control region with the upstream, top and bottom boundaries placed at a distance of 1.5 chord lengths away. For the downstream boundary, a longer distance of three chord lengths from the trailing edge of the section was allocated to ensure the vortices are completely dissipated before leaving the computational domain. The boundary condition types used for upstream, top, bottom and downstream boundaries were velocity inlet, symmetry, symmetry and outflow respectively, whereas the surfaces of the NACA0015 section were designated as no-slip walls.

Discretization of the computational grid was achieved by using quadrilateral cells. As crucial flow features exist within the boundary layer and the viscous sublayer, a denser cell distribution was concentrated near the surface of the two-dimensional section with high cell quality, whereas a coarser cell distribution was gradually extended to the farfield regions to save computational cost. This was achieved through the application of the structured multi-block meshing method, where the entire computational region was divided into 322 four-sided faces with each face independently meshed. Special attention was given to the immediate region aft of the trailing edge where pure orthogonal cells were designated as shown in Figure 2, to maximize the accuracy in capturing the full features of the turbulent and unsteady near wake flows. 


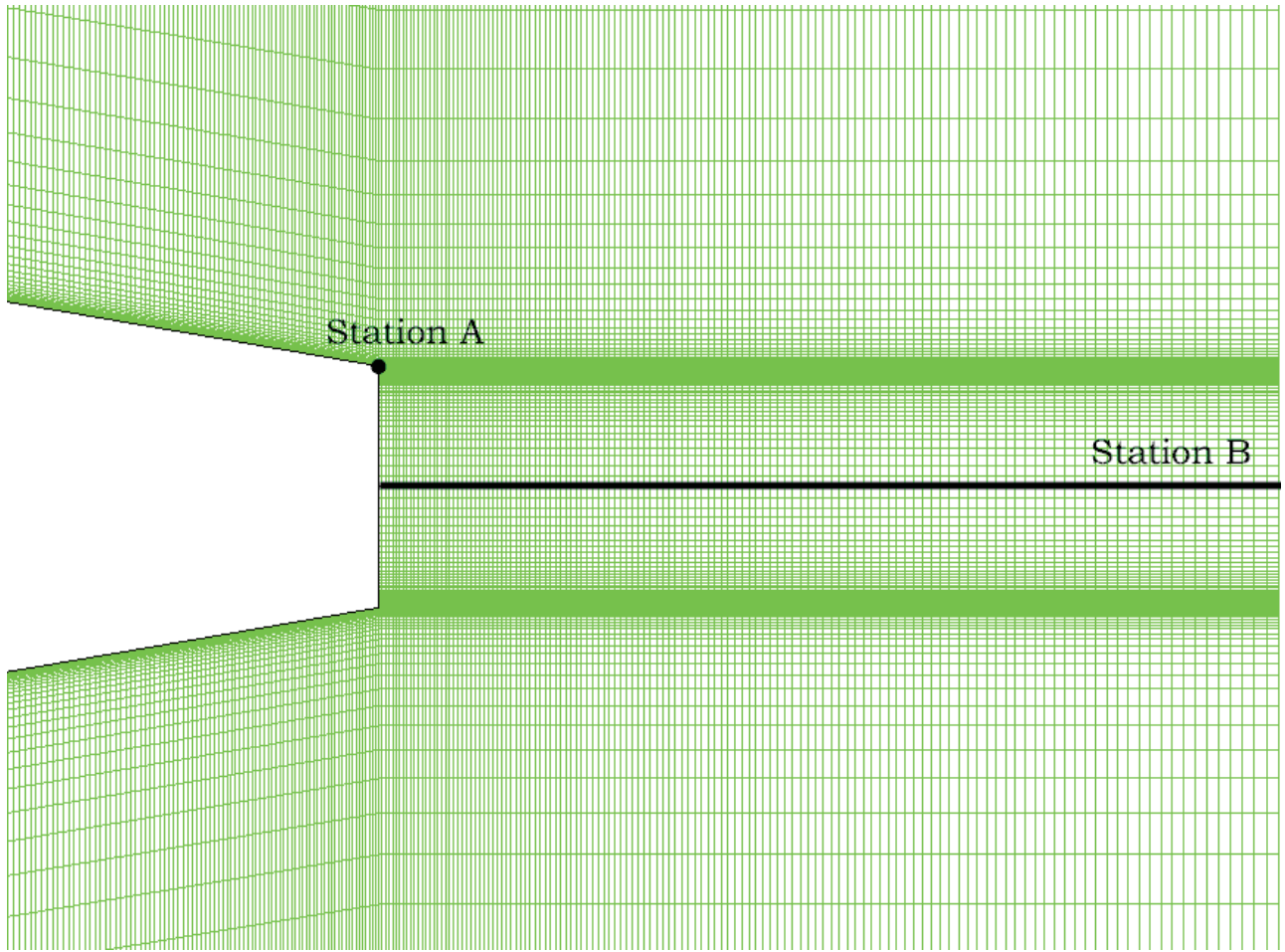

FiguRE 2: Grid distribution at trailing edge and data monitoring stations.

TABLE 1: Summaries of bluntness parameters, shedding frequency and Strouhal number for all models

\begin{tabular}{cccccc} 
Case & $h / \delta^{*}$ & $\mathrm{Re}_{h}$ & Vortex Shedding & $f_{s}(H z)$ & $\mathrm{St}=h f_{s} / U_{\infty}$ \\
\hline $99.8 \% C$ & 0.28 & 13,500 & No & - & - \\
$99.6 \% C$ & 0.34 & 15,900 & No & - & - \\
$99.4 \% C$ & 0.39 & 18,300 & No & - & - \\
$99.2 \% C$ & 0.45 & 20,800 & Yes & 137 & 0.088 \\
$99.0 \% C$ & 0.51 & 23,200 & Yes & 128 & 0.092 \\
$98.8 \% C$ & 0.58 & 25,600 & Yes & 113 & 0.089
\end{tabular}


Six cases were simulated using FLUENT 6.0 (see Table 1). The segregated implicit solver approach was chosen together with the SIMPLE method to achieve pressure-velocity coupling and the field variables were interpolated using the second order upwind scheme. Based on the assessment findings as reported by Mulvany et al. [4], the two-equation Realizable $\kappa-\epsilon$ turbulence model with enhanced wall treatment chosen to resolve the near wall viscous layer. To ensure that the computational flow field had reached a stable condition before subjecting it to unsteady analysis, the simulation was initially processed in steady-state condition, and then switched over to transient computation. Two main data recording stations were designated as shown in Figure 2, with station A recording data for spectral analysis.

\section{Results and Discussion}

Grid independence was performed using the case with cut at the $99.8 \% C$. Five meshes with increasing cell density were developed for analysis, based on the converged solutions of drag coefficient $\left(C_{d}\right)$ and acceptable wall $y^{+}$values $(<1)$. The drag coefficients for five meshes are shown in Figure 3. A converged solution was achieved with Mesh $\mathrm{D}$, which was chosen as the baseline meshing scheme to develop the remaining five cases.

Distribution of time-averaged pressure coefficients $\left(C_{p}\right)$ on the suctionsurface of the airfoil for all six cases is shown in Figure 4 (pressure surface not shown due to symmetry). As the flow progressed up the leading edge curvature, it experienced a favourable pressure gradient region and reached a maximum dynamic pressure at approximately $13.4 \%$ chord. From this point onward the flow entered a region of almost constant adverse pressure gradient up to the trailing edge. Figure 4 also shows that modifying the trailing edge height has virtually no effect on the overall pressure distribution. However, an exploded view of the trailing edge $C_{p}$ distribution consistently shows there is a short region of favourable pressure gradient just before the trailing edge, 


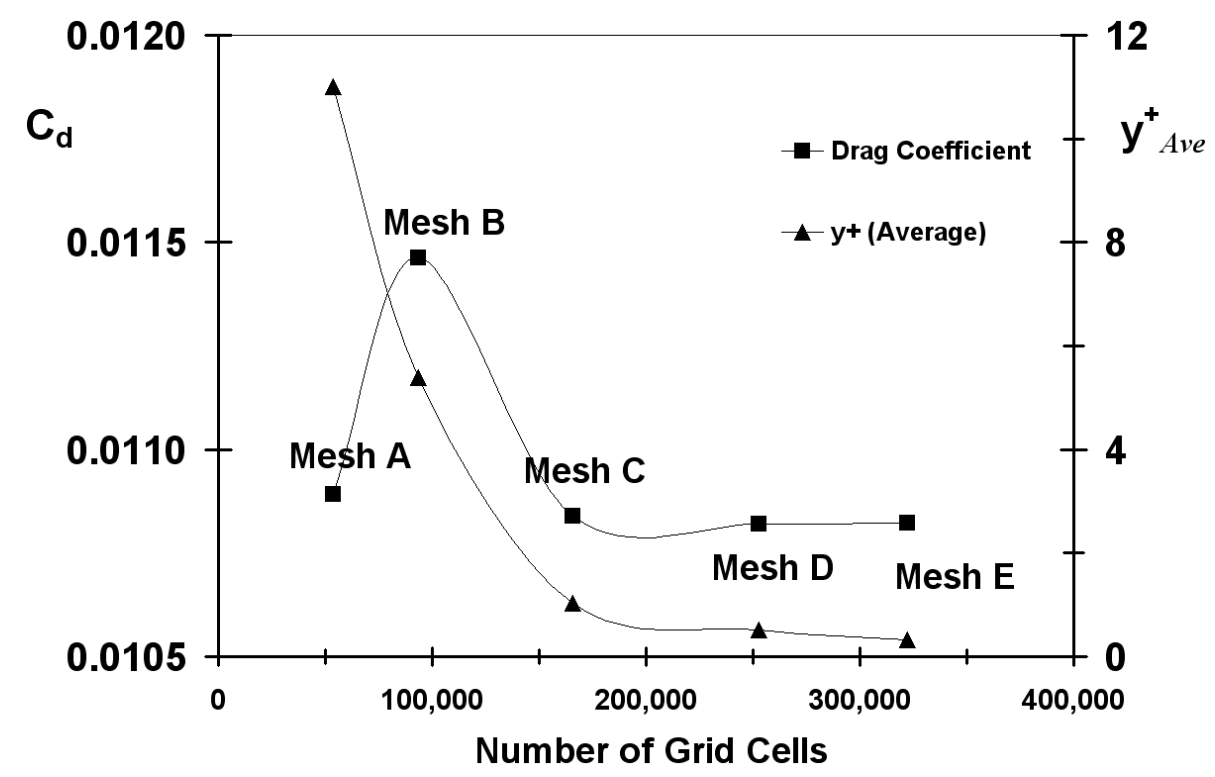

Figure 3: Convergence of drag coefficient and corresponding $y^{+}$values for the $99.8 \% C$ case. 


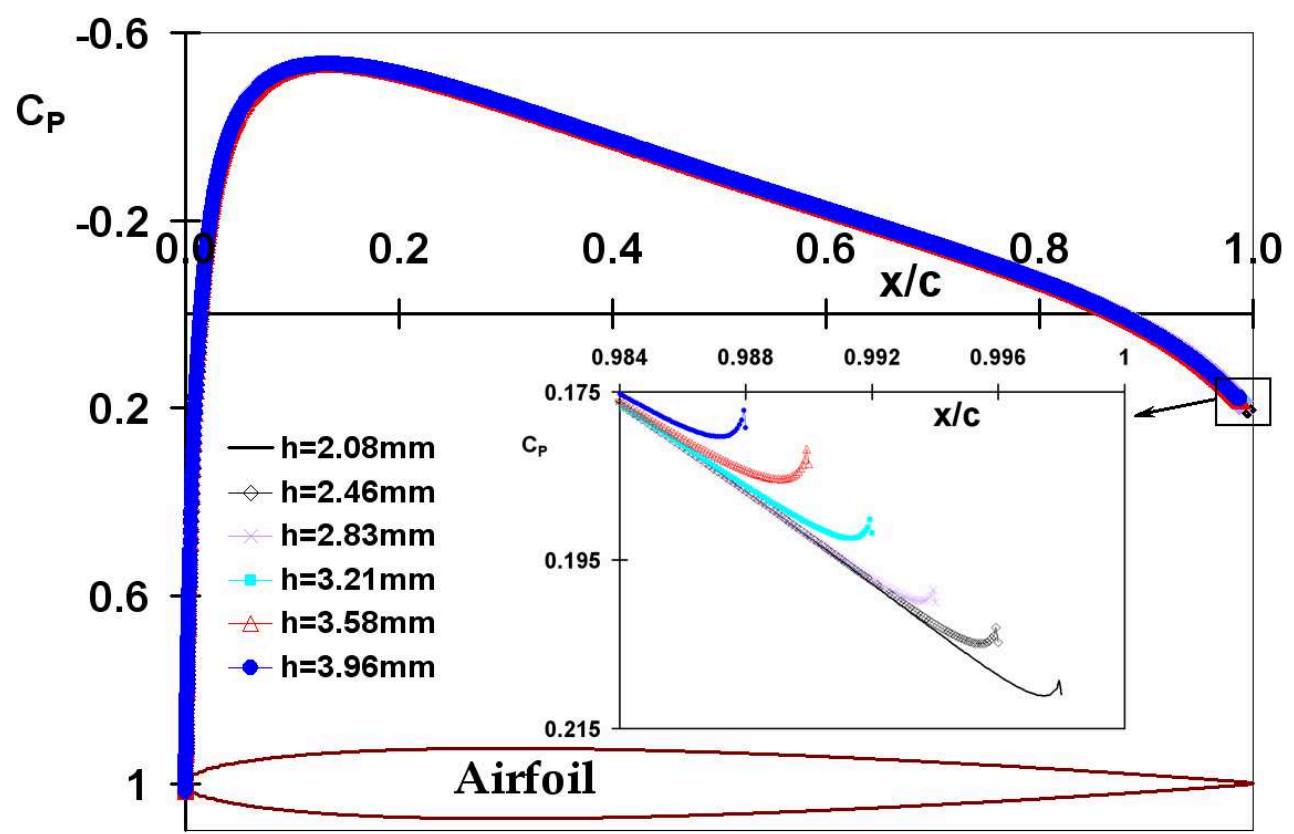

Figure 4: Pressure distribution around the airfoil with different trailing edge heights. 


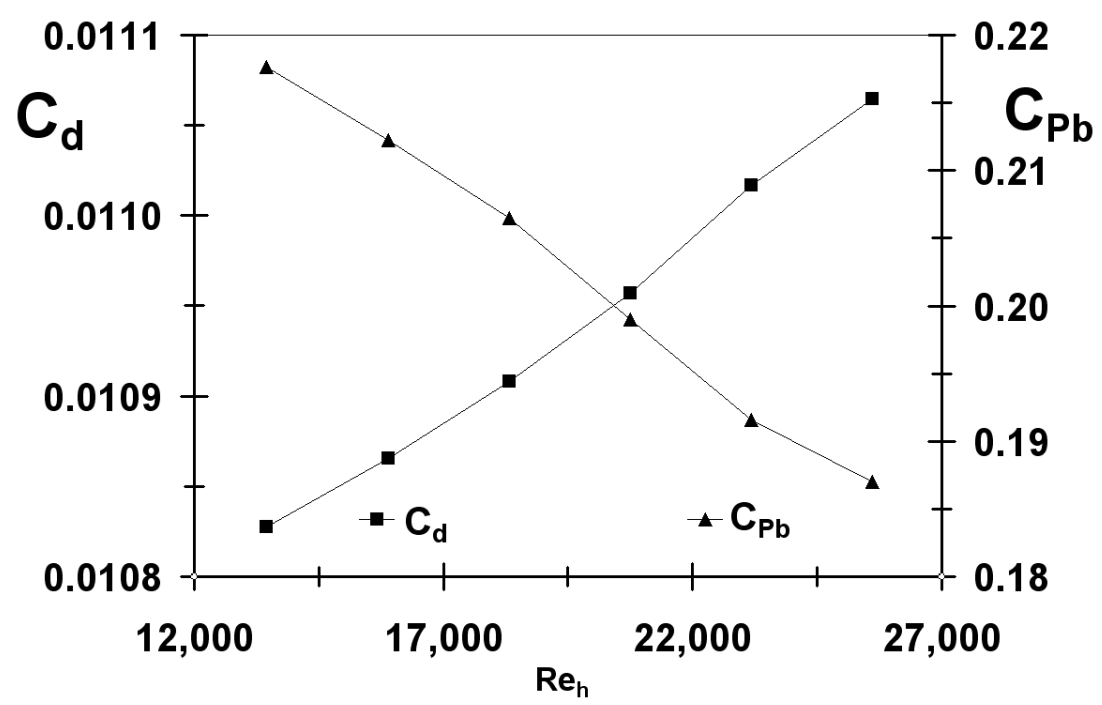

FIGURE 5: Effects of increasing trailing edge height on total drag and back pressure.

which suggests that the flow accelerates locally and hence remain attached to the surface. No early separation was observed for any of the six cases. Moreover, early separation can also be detected by inspecting for locations where the wall shear stress reduces to zero [7], and the analysis of instantaneous wall shear stress distributions about the section for six cases conducted (not shown) further supports no flow separation.

Time-averaged total drag of the section was markedly affected by increasing the trailing edge height as shown in Figure 5. The analysis of the drag contribution sources showed the majority of the drag was due to skin friction $(\geq 73 \%)$. However, the pressure drag was the main cause in the increased total drag coefficient. This was intuitively expected, as by increasing $h$, the immediate wake structure aft of the trailing edge also increases in size producing a growingly larger but lower pressure region. Figure 5 further substantiates this effect as the (normal) base pressure $\left(C_{P b}\right)$ monitored at the 
centre of the vertical trailing edge surface decreased with an increase in $h$ thereby amplifying the pressure offset between the relatively high leading edge stagnation pressure and the lower pressure at the trailing edge.

The computed results from the unsteady state simulation showed that the first three cases did not exhibit vortex shedding, although each case consisted of a circulatory region containing two stationary vortices of the same strength as shown in Figure 6(a). The last three cases, on the other hand, displayed evidence of vortex shedding aft of the trailing edge. Numerical injection of particles enabled flow visualization of the trailing vortices: Figure 6(b) shows a von Karman vortex street wake with fully developed vortices rapidly decaying in vorticity strength as they progressed downstream. Figure 7 shows the time averaged vertical velocity profiles (nondimensionalised with freestream velocity) along the centreline of the section aft of the trailing edge, providing details of how far the vortices take to dissipate downstream of the trailing edge. Close examination showed that stationary vortices affected the local flow field within a distance of approximately $20 \%$ chord length. The moving vortices disturbed a greater distance in the wake flowfield, with the most extreme case $98.8 \% C$ took $70 \%$ chord length aft of the trailing edge to fully dissipate, although the wake disturbance was relatively small reflected by the low magnitudes of the velocity ratios.

The occurrence of vortex shedding caused distinct pressure fluctuations in the local flow field and was illustrated by the power spectra of the data monitored at station A, as shown in Figure 8 for six cases. The dominant peaks correspond to the fundamental vortex shedding frequency $\left(f_{s}\right)$ of each of the last three cases while the less apparent peaks correspond to their respective first harmonics. Figure 8 further supported that no shedding of vortices occurred for the first three cases, as identified previously, and the power spectra suggested that at the same Re, the shedding frequency could be altered by modifying the height of the trailing edge. This has an important implication in situations where the shedding frequency matches the natural frequency of the structure. 


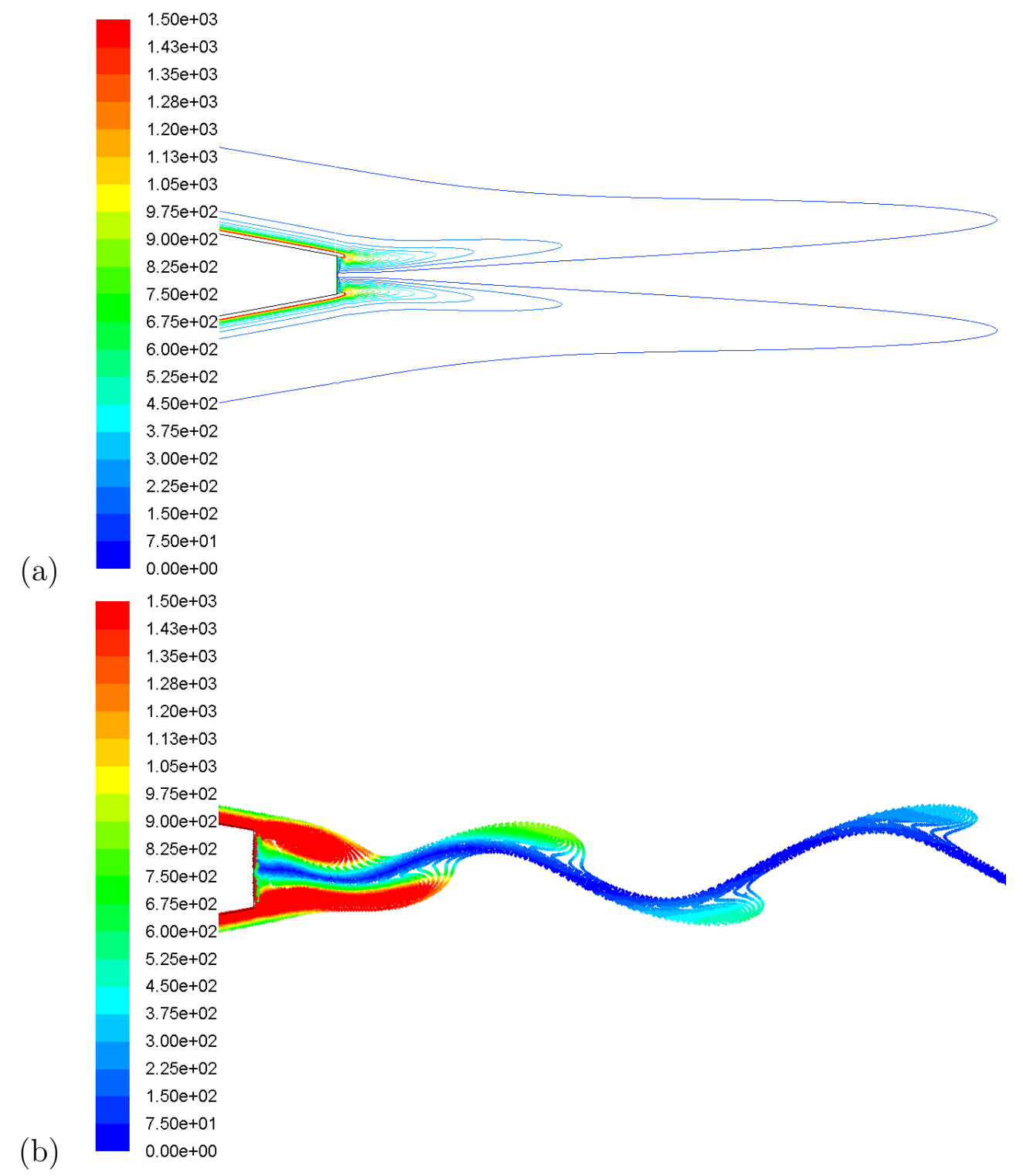

FIGURE 6: Instantaneous vorticity contour for (a) $h=2.83 \mathrm{~mm}$ and (b) $h=3.21 \mathrm{~mm}$ with numerical particle visualization [vorticity magnitude (1/s) colour range: $0-1500]$. 


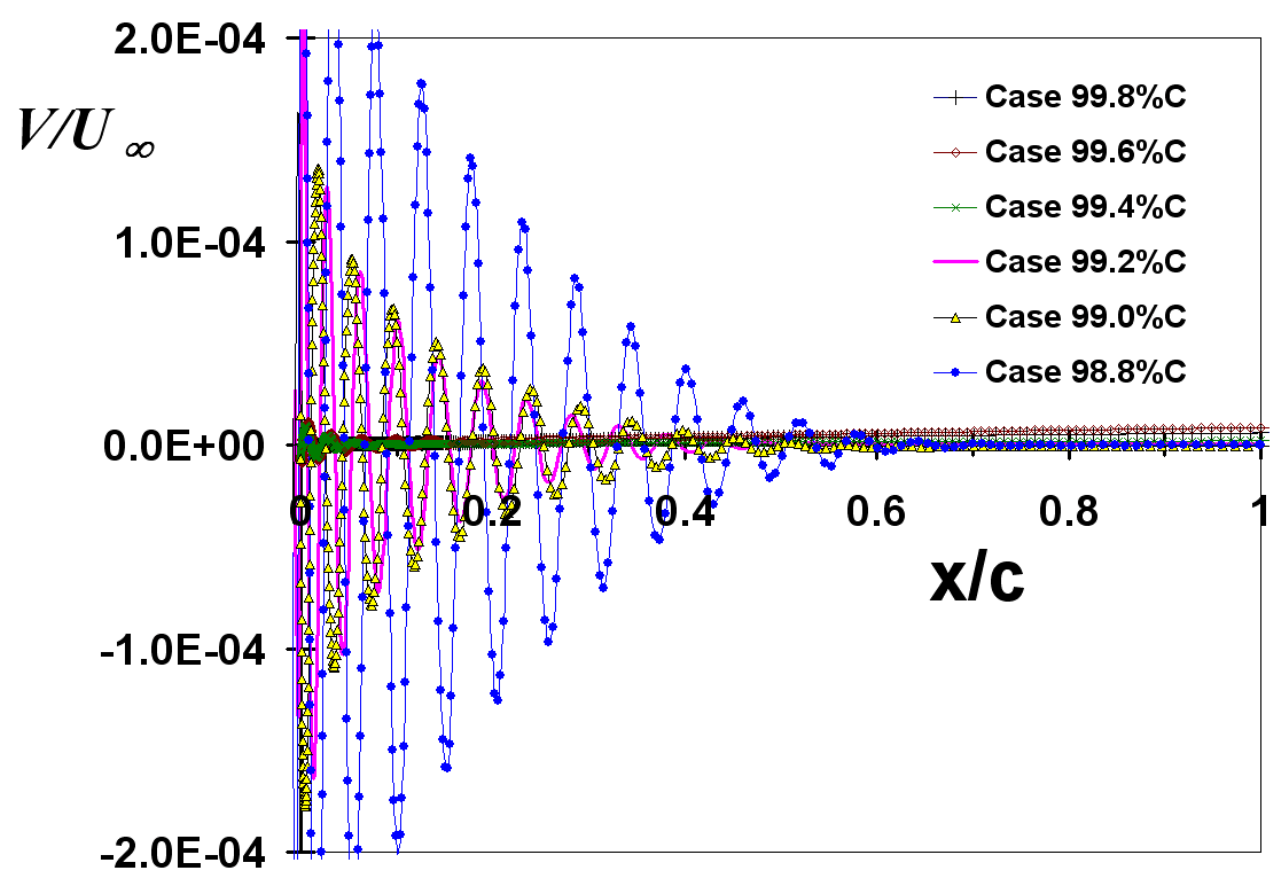

Figure 7: Trailing edge mean velocity profiles for six cases. 


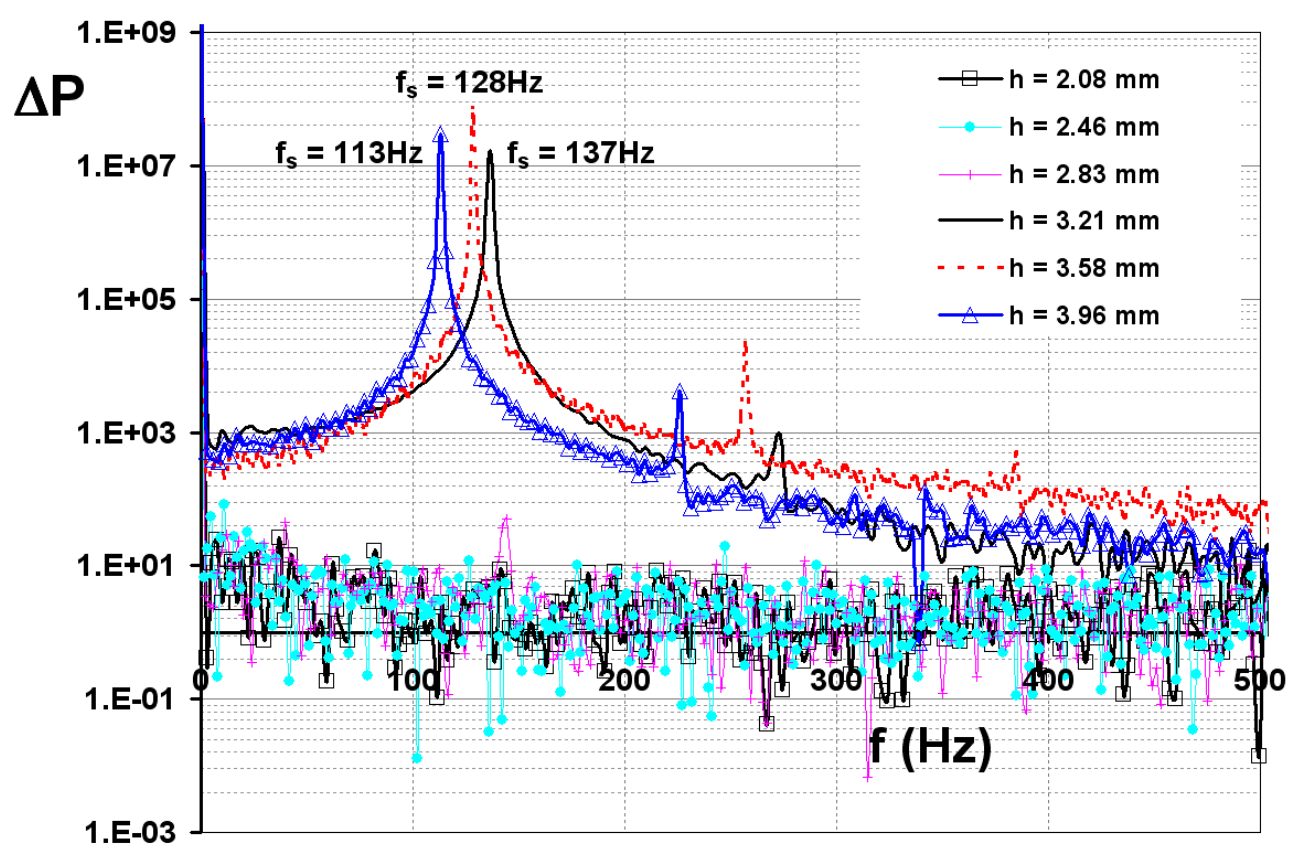

FiguRE 8: Spectra of pressure at station A for different trailing edge heights. 


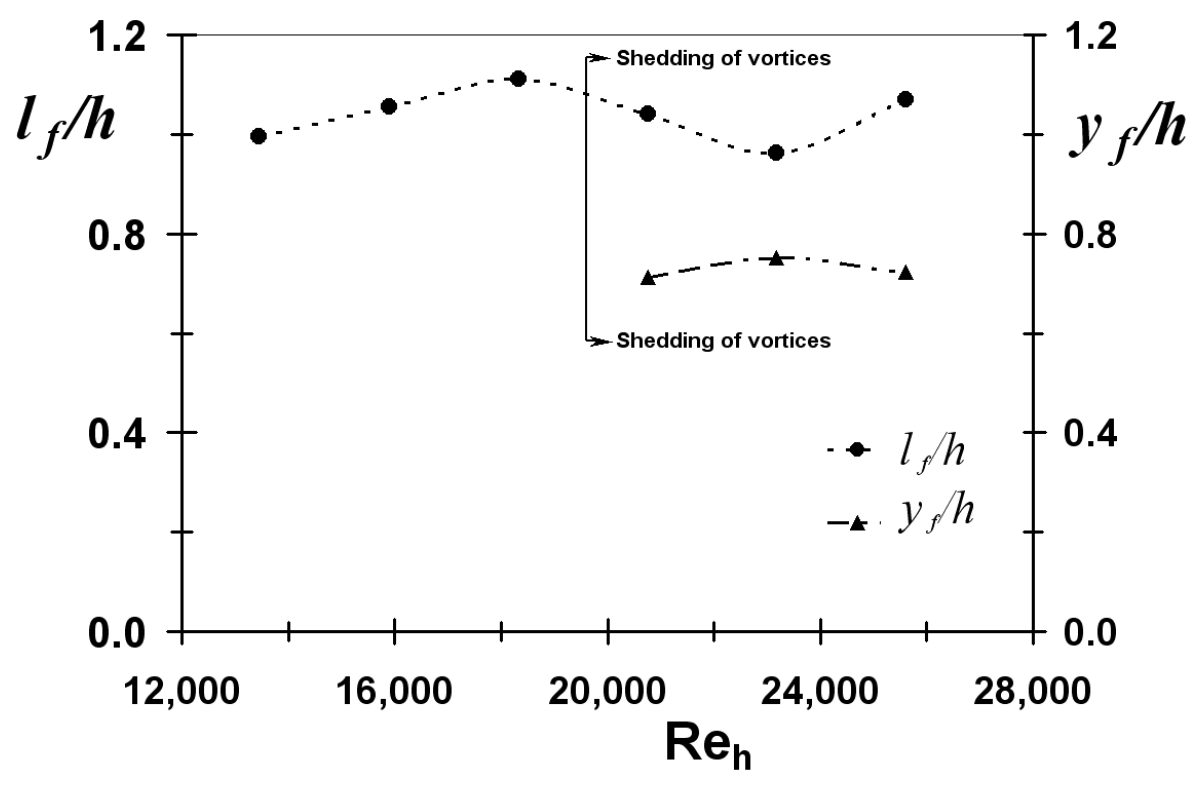

FiguRE 9: Effects of $\mathrm{Re}_{h}$ on wake formation length $l_{f}$ and cross-wake shear layer thickness $y_{f}$.

Table 1 provides a summary of $\mathrm{Re}_{h}$, bluntness parameter, shedding frequency and the Strouhal number for each of the six cases. The necessary degree of bluntness required for vortex shedding to take place was numerically predicted at $h / \delta^{*}=0.45$ with a corresponding $\operatorname{Re}_{h}$ of $2.08 \times 10^{4}$. This is reasonably close to Blake's [1] conclusion that vortices will certainly shed when $h / \delta^{*}>0.50$. Furthermore, Brooks and Hodgeson [6] experimentally found that the bluntness parameter for a closest geometrically similar trailing edge profile, the truncated NACA0012, at a flow with Re of $2.84 \times 10^{6}$, was $h / \delta^{*}=0.48$ but at a lower $\operatorname{Re}_{h}$ of $0.88 \times 10^{4}$. The computed average Strouhal number St $=0.09$ based on the definition shown in Table 1, adapted from Brooks and Hodgeson [6], were also comparable to their finding of $\mathrm{St}=0.1$.

Time-averaged variation of wake formation length $l_{f}$ and cross-wake shear layer thickness $y_{f}$ (taken at $l_{f}$ ) with increasing $\mathrm{Re}_{h}$ are shown in Figure 9. 
$y_{f}$ was found to change negligibly with $\mathrm{Re}_{h}$ and attained an average value of approximately $y_{f} / h=0.73$. This numerical result was half of the value reported by Blake [1] with $y_{f} / h=1.5$ for the NACA0012 section, which was calculated from the experimental data performed by Brooks and Hodgeson [6], but was not surveyed experimentally. This (calculated) high value is questionable as $y_{f}$ is defined by the distance between the two local maxima of the velocity fluctuations caused by the shed vortices [8] and for a symmetrical body, Bearman [8] consistently reported that the distance between these loci are approximately equal to or shorter than the trailing edge height, up to the end of the vortex formation region $l_{f}$. Variation of $l_{f}$ on the other hand, was observed to exhibit a similar trend to that of flow past a circular cylinder $[9,1]$ both before and after the onset of vortex shedding up to $\operatorname{Re}_{h}=23,200$ (case $99.0 \% C$ ). At $\operatorname{Re}_{h}=25,600$ formation length was again elongated, which was not expected and hence requires further numerical investigation and experimental validation.

\section{Conclusion}

The various unsteady trailing edge flow characteristics have been successfully predicted using the unsteady RANS approach with reasonable agreement to experimental data. The onset of vortex shedding was numerically determined at $h / \delta^{*}=0.45$ compared to the published data of 0.50 [1] and 0.48 [6]. In addition, the computed Strouhal numbers for vortex shedding cases were reasonably close to the existing experimental results [6] for a similar trailing edge profile. Furthermore, we found that at the same Reynolds number, variation of the degrees of the trailing edge bluntness has critical effects on the flow characteristics. Firstly, by increasing the trailing edge height, the shedding of the vortices reduces in frequency. Secondly, the majority of the wake formation length variation with $\mathrm{Re}_{h}$ resembles that of flow past a circular cylinder, although the last case needs further verification. Finally, the Strouhal number remains fairly constant with $\mathrm{Re}_{h}$, attaining an averaged 
value of 0.09 for this blunt NACA0015 section.

\section{References}

[1] W. K. Blake, Mechanics of Flow-Induced Sound and Vibration, Vols. I and II, Academic Press, Orlando, 1986, (Chapters 4 and 11). C824, C825, C836, C837

[2] D. A. Bourgoyne, S. L. Ceccio, D. R. Jessup, S. Park, J. Brewer and W. R. Pankajakshan, Hydrofoil Turbulent Boundary Layer Separation at High Reynolds Numbers, 23rd Symposium on Naval Hydrodynamics, Val de Reuil, France, 2000.

http://me.engin.umich.edu/hifoil/Papers/NavyHydro2000.pdf C824

[3] J. S. D. Ostertag, A. Celic and S. Wagner, Trailing-Edge Noise Prediction By SATIN On The Basis of Steady RANS Solutions, 8th AIAA/CEAS Acoustics Conference and Exhibition Agenda, Breckenridge, Colorado, 17-19 June 2002. http:

//pdf.aiaa.org/preview/CDReadyMAER002_554/PV2002_2471.pdf C825

[4] N. Mulvany, J. Tu, L. Chen and B. Anderson, Assessment of Two-Equation Turbulence Modelling for High Reynolds Number Hydrofoil Flow, Int. J. Numer. Meths. Fluids, 45, 2004, pp.275-299. doi:10.1002/fld.698 C825, C828

[5] D. Ang, L. Chen and J. Tu, Unsteady RANS Simulation of High Reynolds Number Trailing Edge Flow, Proceedings of 15th Australasian Fluid Mechanics Conference, Sydney, Australia, 2004. http://www. aeromech. usyd.edu.au/15afmc/proceedings/papers/ AFMC00161.pdf C825 
[6] T. F. Brooks and T. H. Hodgeson, Trailing Edge Noise Prediction Using Measured Surface Pressures, Journal of Sound and Vibration, 78, 1981, pp.69-117. doi:10.1016/S0022-460X(81)80158-7 C825, C836, C837

[7] H. Schlichting, Boundary Layers, 7th Edition, McGraw-Hill, 1979. C831

[8] P. W. Bearman, Investigation of the Flow Behind a Two-Dimensional Model with a Blunt Trailing Edge and Fitted with Splitter Plates, Journal of Fluid Mechanics, 21, part 2, 1965, pp.69-117. doi:10.1017/S0022112065000162 C837

[9] S. M. Bloor, The transition to turbulence in the wake of a circular cylinder, Journal of Fluid Mechanics, 19, 1964, pp.290-304. doi:10.1017/S0022112064000726 C837 